\title{
Irreducible posterolateral dislocation of the knee: a case report
}

\author{
GIUSEPPE SOLARINO, ANGELA NOTARNICOLA, GIUSEPPE MACCAGNANO, \\ ANDREA PIAZZOLLA, BIAGIO MORETTI
}

Department of Basic Medical Sciences, Neuroscience and Sense Organs, Orthopaedic Unit, University of Bari

"Aldo Moro", Bari, Italy

\begin{abstract}
Irreducible posterolateral dislocations of the knee are rare lesions, generally caused by high-energy trauma inducing rotational stress and a posterior and lateral displacement of the tibia.

In these conditions, the interposition of abundant soft tissue inside the enlarged medial joint space prevents spontaneous reduction or non-surgical treatment by manipulation of the dislocation. Surgical treatment is therefore compulsory.

We report the clinical case of a woman who suffered a subluxation of the knee while jogging. The case we describe is of interest because it shows that even less severe knee dislocations, like this subluxation caused by a low-velocity sports trauma, may present in an irreducible form requiring open surgery. Clinicalinstrumental monitoring did not reveal any signs of vascular or nerve injury. Owing to the irreducibility of the lesion we were obliged to perform open surgery in order to free the joint from the interposed muscle tissue and repair medial capsule-ligament lesions. Repair of the damaged cruciate ligaments was deferred to a second stage, but ultimately rendered necessary by the persistence of joint instability and the need to address the patient's functional needs. In the literature, different one- and two-step surgical options, performed by arthroscopy or arthrotomy, are reported for such related problems.

The Authors discuss these various options and exami-
\end{abstract}

\section{Corresponding Author:}

Angela Notarnicola, PhD, MD

Department of Basic Medical Sciences, Neuroscience and

Sense Organs, Orthopaedics Unit, University of Bari

"Aldo Moro"

Piazza G. Cesare 11, 70124 Bari, Italy

E-mail: angela.notarnicola@uniba.it ne and discuss their own decision taken during the surgical work-up of this case.

Key Words: knee, posterolateral, dislocation, anterior cruciate ligament, injury.

\section{Introduction}

Dislocation of the knee is a rare orthopaedic emergency that requires appropriate monitoring and treatment. It has a low estimated annual incidence, accounting for less than $0.01 \%$ of trauma episodes, even if the true number of cases is unknown because many dislocations reduce spontaneously already at the site of the traumatic event (1). Most lesions of this kind are caused by high-velocity sports traumas (2), in which traumatic forces produce stress in the sagittal plane of the knee in the anteroposterior direction and in the frontal plane in varus-valgus angulation (3), inducing a rotational dislocation of the tibia in relation to the femoral condyles.

This causes lesions of the ligament structures, also involving the popliteal artery in $20 \%$ of cases and the common peroneal or tibial nerve in $19 \%$ (4). In the literature, the role of non-surgical "closed" reduction is emphasized (5), while surgical treatment should be reserved for irreducible cases or those in which vesselnerve complications arise or there are signs of immediate joint instability (6).

\section{Case report}

A healthy 51-year-old woman suffered a traumatic sprain of her left knee when she fell while jogging. Unable to stand or walk, she was immediately transfer- 
red by ambulance to the emergency department of our hospital where the clinical evaluation showed the left knee to be fixed in slight flexion by $10^{\circ}$ and valgus by $5^{\circ}$, as compared to the contralateral joint, as well as showing severe ecchymosis with invagination of skin and soft tissue on the medial side, a presentation defined as the "dimple sign" (7) (Fig. 1).

Tests for ligament laxity were difficult to perform due to the patient's intense pain, which was exacerbated by palpation and manual compression; there was no peripheral (sensory and motor) nerve damage, and the distal vascular arteries (both the posterior tibial artery and the dorsal pedis artery) pulsed as normal.

Knee radiographs performed in anteroposterior and lateral views showed narrowing of the lateral femorotibial articular space and alignment of the femoral cortex anterior to the tibial shin (Fig. 2).

Both clinical and radiological tests were suggestive of posterolateral dislocation of the knee and therefore digital femoral arteriography was performed to exclude any damage to the popliteal artery (Fig. 3).

Computed tomography (CT) scans of the knee joint showed complete rupture of the anterior cruciate ligament (ACL) and of both the medial and the lateral menisci, but no osteochondral injuries (Fig. 4).

The patient was admitted to our orthopaedic department and overnight, within a few hours of the trauma, an attempt to reduce the dislocation was made in the operating room, under sedation.

The reduction maneuver, performed by pushing the

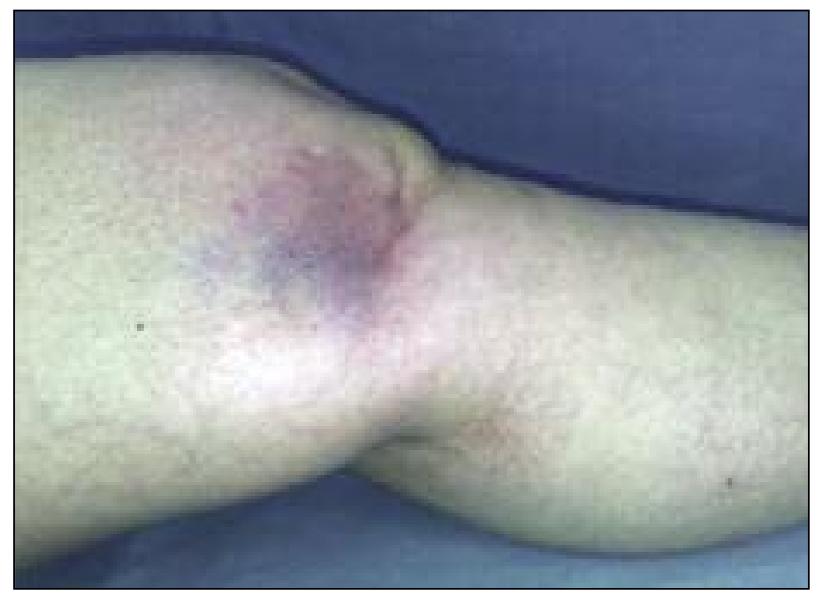

Fig. 1. Clinical appearance of the left knee in slight flexion and valgus, with invagination and severe ecchymosis of the skin and soft tissue on the medial side ("dimple sign").
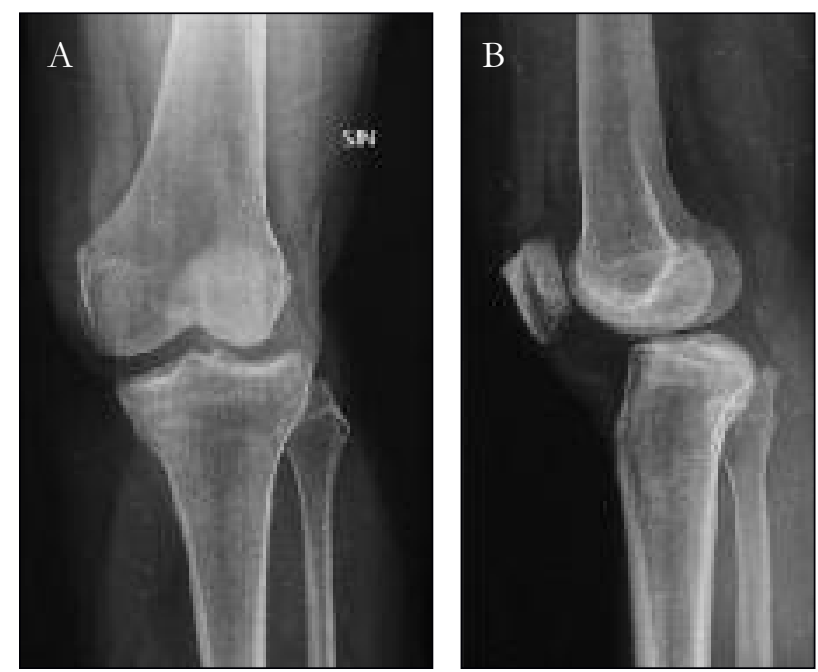

Fig. 2. Anteroposterior (A) and lateral (B) X-rays of the left knee after the trauma show a posterolateral subluxation of the tibia and enlargement of the medial joint space.

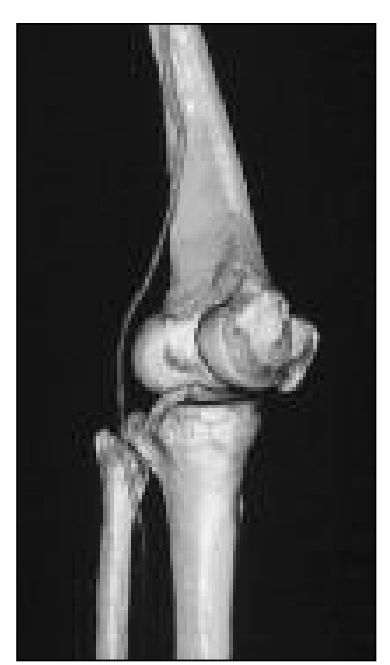

Fig. 3. The digital femoral arteriography excluded any damage of the popliteal artery.

distally to the lower border of the pes anserinus.

Reverting the skin and the subcutaneous fat flap posteriorly, the medial femoral condyle was evident through an ample breach of the extensor retinaculum, the deep fascia and the capsule, all invaginated inside the joint space (Fig. 5).

There appeared to be a complete rupture of the medial collateral ligament (MCL), a dislocation of the pes anserinus at the muscle-tendon junction, and a detachment of the femoral insertion of the medial gastrocnemius muscle (Fig. 6). Both the ACL and 


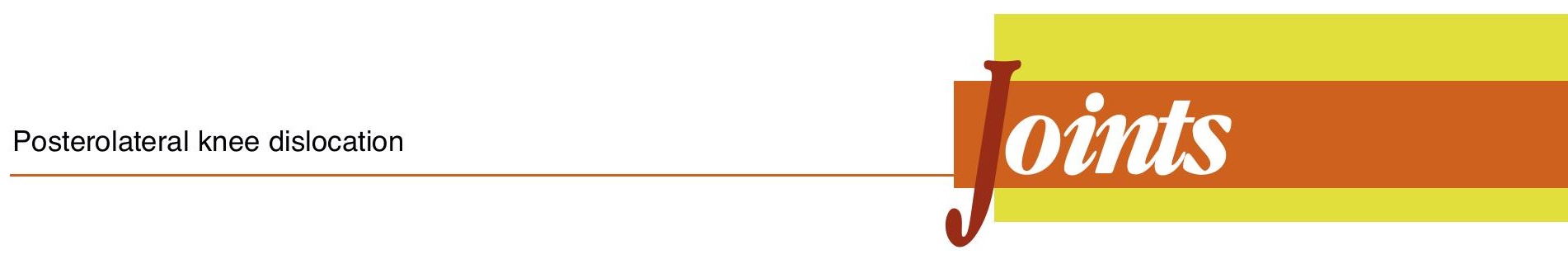

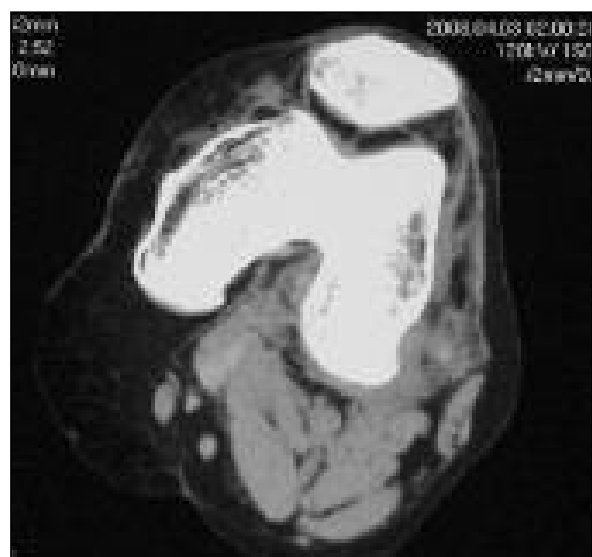

Fig. 4. CT scan showing complete rupture of the anterior cruciate ligament, but no osteochondra injuries.

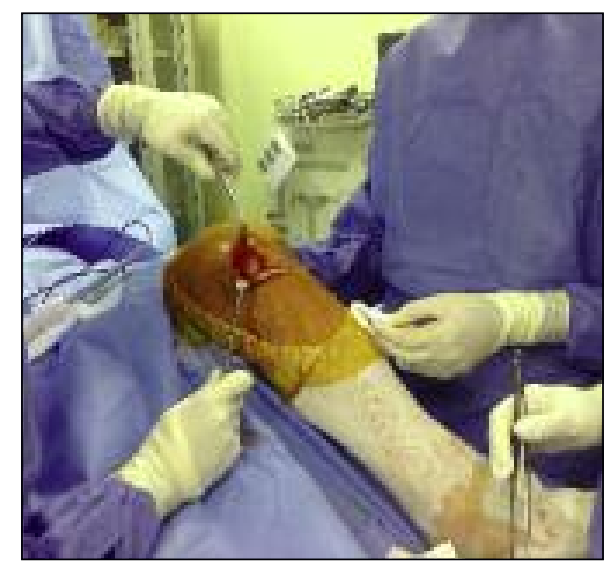

Fig. 5. The medial femoral condyle was evident through an ample breach of the extensor retinaculum, the deep fascia and the capsule.

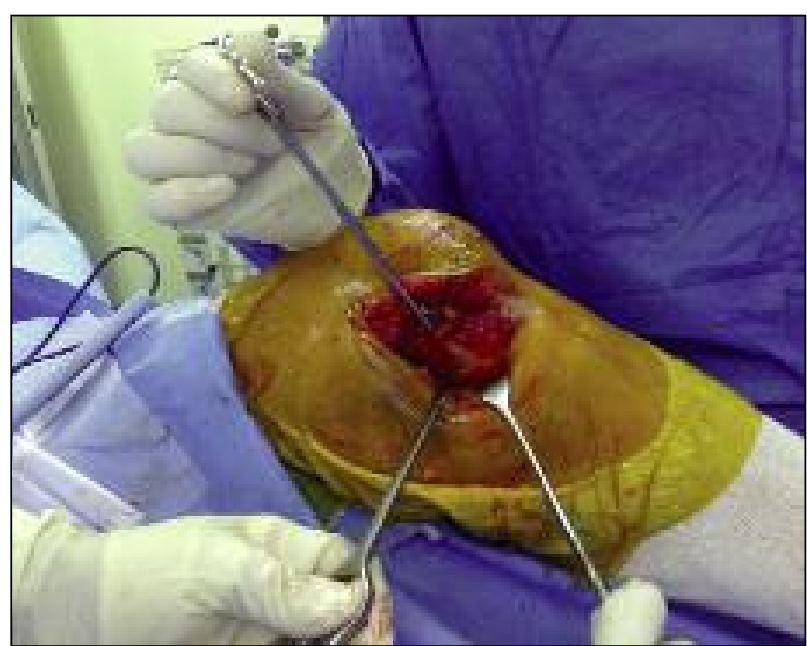

Fig. 6. Complete rupture of the MCL. The proximal end of the ligament is held by the scissors.

posterior cruciate ligament (PCL) were torn. Medial meniscus was intact.

After reduction of the pes anserinus, the correct relations between the joint components appeared to be restored; the medial gastrocnemius muscle and the MCL were reinserted proximally at their origin on the femur with three suture anchors (Fig. 7) and the capsular breach was sutured with interrupted \#1 absorbable braided sutures. In view of the high risk of recurrence of the dislocation in the immediate post-traumatic period, it had been decided during the pre-surgical workup that repair of the damaged cruciate liga- ments with a tendon autograft would be deferred, also because the pes anserinum tendon had been traumatized. The skin was closed with interrupted mattress sutures and an above-the-knee plaster was applied with the joint flexed to about $90^{\circ}$.

The patient was discharged on the fourth postoperative day, with instructions to avoid weight bearing for six weeks. At the end of this period, a rehabilitation program was started to restore the joint function and muscle strength and complete weight bearing was allowed after a further two weeks.

Regular follow-up visits were scheduled. At the 18months of follow-up, although complete recovery of the range of motion (ROM) was demonstrated, and good muscle recovery, the patient continued to complain of a feeling of joint instability.

To address her functional needs she was therefore submitted to arthroscopic reconstruction of the ACL, using as grafts the semitendinous and gracilis tendons fixed with an EndoButton CL (Smith and Nephew, Memphis, TN, USA) on the femoral side and with a poly-L-lactic acid biodegradable interference screw on the tibia.

At the last follow-up, one year after the second procedure and two and a half years after the trauma, the patient's knee showed no signs of instability nor functional limitation of the joint, and ROM of $0-120^{\circ}$ was restored. Magnetic resonance performed at one year after ACL reconstruction showed good graft incorporation (Fig. 8).

\section{Discussion}

As proposed by Kennedy (9), knee dislocations are classified, according to the direction of tibial dislocation in relation to the femur, as anteromedial, posteromedial, anterolateral and posterolateral. In our experience, supported by a thorough literature search, many of the irreducible cases are posterolateral dislocations, 


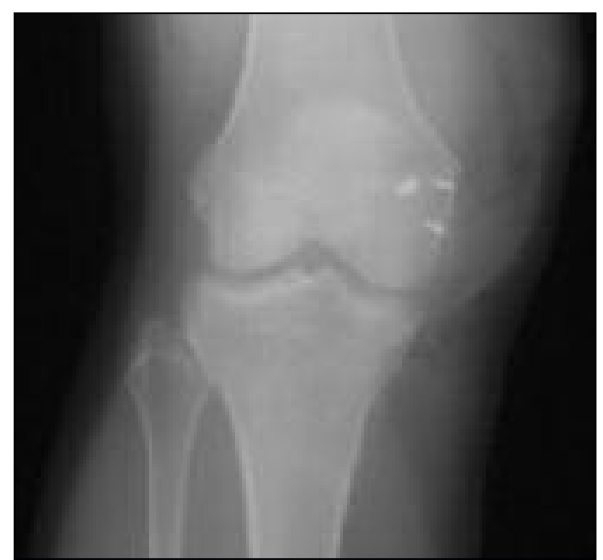

Fig. 7. Postoperative radiograph showing the three suture anchors used to fix the medial gastrocnemius muscle and the MCL proximally.

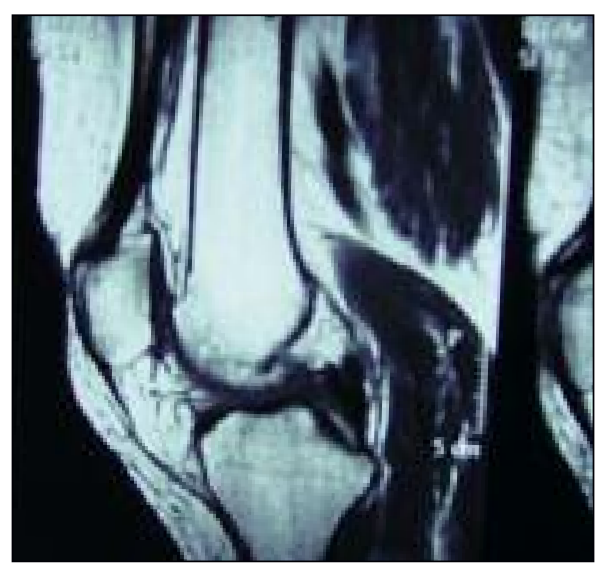

Fig. 8. MR at 1 year after the arthroscopic reconstruction of the ACL showed good retention of the fixation devices and satisfactory healing of the graft. in which the medial retinaculum and capsule are interposed in the joint space (5-10). The traumatic mechanism, which causes excessive strain on the knee joint in slight flexion and valgus with tibial external rotation and internal rotation of the femur (3), generally occurs during high-velocity sporting activities, such as skiing, surfing, mountain biking, car racing, etc., that are at higher risk for these lesions (7-11). In these cases, the interposition of abundant soft tissue inside the enlarged joint space prevents reduction of the dislocation and surgery becomes necessary. Different surgical procedures have been described in the literature, each yielding satisfactory results. Dislocation can be reduced arthroscopically or by open surgery and ligament repair can be performed immediately or deferred to a later phase, while an immobilizing plaster cast is generally positioned, although in some cases external fixation is preferred (12-14).

The case we reported is of interest because it describes an irreducible subluxation of the knee caused by a low-impact sports trauma, which resulted in posterolateral tibial dislocation and in lesions of the ACL, PCL, MCL and lateral meniscus. The first point to consider is that even mild traumatic events can cause lesions as severe as those induced by high-velocity trauma (14). Moreover, it should be noted that CT scanning was unable to provide an accurate picture of the damage due to the concomitant presence of abundant post-traumatic intra-articular blood-serum leakage (15). Although older studies suggested a non-surgical option for knee dislocations, a recent meta-analysis is crucial when evaluating the prognosis; in fact, lesions of the popliteal artery or peroneal and tibial nerve require immediate surgery (16-18). In the case we described, the patient did not show any symptoms of vascular or nerve damage, in agreement with literature reports of an incidence of such complications of only $4.8 \%$ in dislocations secondary to low-velocity traumas (19) versus 65\% after high-velocity traumatic events (20).

Meyers and Harvey (20) stressed the rarity of knee dislocations: indeed, an orthopaedic surgeon during his/her professional practice may never be called upon to manage such a trauma. Assessment of the severity of the dislocation, complete or incomplete $(21,22)$, and of the nature of the accident, high or low velocity (23), is not predictive of the risk of irreducibility, which occurs in 4\% of all knee dislocations (24). In such cases, the orthopaedic surgeon must weigh up the advantages of proceeding with acute or deferred surgical reduction, the latter needing to be performed within the shortest possible time, to minimize the risk of onset of complications such as necrosis of the soft tissues, or the compartment syndrome $(24,25)$.

Most knee dislocations could be reduced under sedation by closed methods, but a small proportion are irreducible and require urgent surgical intervention (26); the impossibility of reducing the joint is due mainly to incarceration of all the capsule-ligament structures in the intercondylar notch with the medial condyle in an extra-articular position (24). In a few cases, interposition of the muscles (principally the 
vastus medialis) between the femoral condyles has been reported (27).

There is still no consensus as to the best surgical method, and treatment options range from arthroscopic debridement of the capsule-ligament structures (28) to open reduction and removal of the muscle interposition $(14,29-31)$. In cases of muscle dislocation different surgical choices are advocated: sectioning of the muscle to allow reduction of the knee dislocation (27) or repair of the muscle rupture to return the muscle to its anatomical site (29).

Durakbaşa et al. reported that the medial meniscus may often be detached from the meniscocapsular junction and in these cases it is necessary to extract the medial meniscus from the joint to reduce the dislocation (32).

In the case we reported, open surgical treatment allowed us to reduce the dislocation of the pes anserinus and of the medial gastrocnemius muscle and to repair medial capsule-ligament lesions, while there were no injuries of the medial meniscus needing to be repaired. In the literature, there is no universal agreement as to whether and when lesions of the cruciate ligaments should be repaired.

A one-step reduction method combining arthroscopic cruciate ligament reconstruction is described $(29,33)$. In this kind of lesion, Bistolfi et al. (29) confirmed that lateral and rotational stability must be achieved immediately by reconstruction or suture of the peripheral structures, while reconstruction of the cruciate ligaments can be postponed and even avoided in elderly patients or those not professionally engaged in high level sports. In view of reports of cases of redislocation of the knee immediately after reconstruction of the central pivot (26), we opted, in our case, to treat initially only the MCL, leaving out the ACL and PCL. Other Authors have described a two-step surgical procedure, generally consisting of a first arthroscopic debridement of soft tissues followed by second open stage to suture damaged ligaments (33).

In accordance with the literature $(14,26)$, we hypothesized that the initial instability could compromise the reconstruction of the cruciate ligaments and therefore decided to treat the latter in a second step.

At subsequent clinical follow-up the signs of instability prompted us to decide, in complete agreement with the patient, to repair the ACL. Our decision was supported by the patient's subsequent good recovery and complete return to daily activities, at the same level as prior to the trauma.

In conclusion, the reported case demonstrates that even incomplete dislocations of the knee caused by low-impact trauma can present clinical pictures that are difficult to treat and nearly always require surgery. In view of the risk of onset of vessel-nerve complications, close clinical-instrumental monitoring is essential in the early hours. In cases where reduction cannot be otherwise obtained, surgery is the only solution to ensure the restoration of correct relations between the joint components and, above all, to prevent later recurrences or joint instability.

Complete reduction of the dislocation should be the first aim of treatment, while the opportuneness of proceeding with reconstruction of the cruciate ligaments should be evaluated at a later stage, depending on the functional picture and the patient's activities and requirements.

\section{References}

1. Schenck RC Jr. The dislocated knee. Instr Course Lect. 1994;43:127-136.

2. Wascher DC. High-velocity knee dislocation with vascular injury. Treatment principles. Clin Sports Med. 2000;19:457477.

3. Urgüden M, Bilbaşar H, Ozenci AM, Akyildiz FF, Gür S. Irreducible posterolateral knee dislocation resulting from a low-energy trauma. Arthroscopy. 2004;20 (Suppl 2): 50-53.

4. Wascher DC, Dvirnak PC, DeCoster TA. Knee dislocation: initial assessment and implications for treatment. J Orthop Trauma. 1997;11:525-529.

5. Nystrom M, Samimi S, Ha'Eri GB. Two cases of irreducible knee dislocation occurring simultaneously in two patients and a review of the literature. Clin Orthop Relat Res. 1992; (277):197-200.

6. Liow RY, McNicholas MJ, Keating JF, Nutton RW. Ligament repair and reconstruction in traumatic dislocation of the knee. J Bone Joint Surg Br. 2003;85:845-851.

7. Harb A, Lincoln D, Michaelson J. The MR dimple sign in irreducible posterolateral knee dislocations. Skeletal Radiol. 2009;38:1111-1114.

8. Slocum DB, Larson RL, James SL. Late reconstruction of ligamentous injuries of the medial compartment of the knee. Clin Orthop Relat Res. 1974;(100):23-55.

9. Kennedy JC. Complete dislocation of the knee joint. J Bone Joint Surg Am. 1963;45:889-904.

10. Huang FS, Simonian PT, Chansky HA. Irreducible posterolateral dislocation of the knee. Arthroscopy. 2000;16:323-327.

11. Harner CD, Waltrip RL, Bennett CH, Francis KA, Cole B, Irrgang JJ. Surgical management of knee dislocations. J Bone Joint Surg Am. 2004;86-A:262-273.

12. Chhabra A, Cha PS, Rihn JA, Cole B, Bennett CH, Waltrip RL, et al. Surgical management of knee dislocations. Surgical 


\section{oints}

technique. J Bone Joint Surg Am. 2005;87 Suppl 1:1-21.

13. Ohkoshi Y, Nagasaki S, Shibata N, Yamamoto K, Hashimoto T, Yamane S. Two-stage reconstruction with autografts for knee dislocations. Clin Orthop Relat Res. 2002;(398):169-175.

14. Siegmeth A, Menth-Chiari WA, Amsuess H. A rare case of irreducible knee dislocation in a seventy-three-year-old male. J Orthop Trauma. 2000;14:70-72.

15. Tsiagadigui JG, Sabri F, Sintzoff S, Schuind F. Magnetic resonance imaging for irreducible posterolateral knee dislocation. J Orthop Trauma. 1997;11:457-460.

16. Dedmond BT, Almekinders LC. Operative versus nonoperative treatment of knee dislocations: a meta-analysis. Am J Knee Surg. 2001;14:33-38.

17. Klineberg EO, Crites BM, Flinn WR, Archibald JD, Moorman CT 3rd. The role of arteriography in assessing popliteal artery injury in knee dislocations. J Trauma. 2004;56:786-790.

18. Gruber H, Peer S, Meirer R, Bodner G. Peroneal nerve palsy associated with knee luxation: evaluation by sonography--initial experiences. AJR Am J Roentgenol. 2005;185:1119-1125.

19. McCoy GF, Hannon DG, Barr RJ, Templeton J. Vascular injury associated with low-velocity dislocations of the knee. J Bone Joint Surg Br. 1987;69:285-287.

20. Meyers MH, Harvey JP Jr. Traumatic dislocation of the knee joint. A study of eighteen cases. J Bone Joint Surg Am. 1971;53:16-29.

21. Curtin W, O'Farrell D, Dolan M, O'Brien T. An irreducible posterolateral knee subluxation. Ir J Med Sci. 1994;163:459460.

22. Saini R, Mootha AK, Goni VG, Dhillon MS. Neglected irreducible posterolateral knee dislocation. Indian J Orthop. 2010;44:468-470.

23. Schaefer RA, Bellafiore VA, Corzatt RD. Irreducible dislocation of the knee. Mil Med. 1999;164:827-829.

24. Chirpaz-Cerbat JM, Rossi J, Mélère G, Martinez T.
Irreducible knee dislocation by medial capsulo-ligament incarceration. Rev Chir Orthop Reparatrice Appar Mot. 2004;90:449-455.

25. Back DA, Rauhut F, Rieger H. Knee dislocation - a simple diagnosis? Compartment syndrome with occlusion of the popliteal artery and lesion of the peroneal nerve after inadequate trauma. Unfallchirurg. 2011;114:66-69.

26. Gu MQ, Deng L, Liu Y. Posterolateral dislocation of the knee joints: analysis of 9 cases. Chin J Traumatol. 2004;7:210216.

27. Silverberg DA, Acus R. Irreducible posterolateral knee dislocation associated with interposition of the vastus medialis. Am J Sports Med. 2004;32:1313-1316.

28. Said HG, Learmonth DJ. Chronic irreducible posterolateral knee dislocation: two-stage surgical approach. Arthroscopy. 2007;23:564.e1-4.

29. Bistolfi A, Massazza G, Rosso F, Ventura S, Cenna E, Drocco $\mathrm{L}$, et al. Non-reducible knee dislocation with interposition of the vastus medialis muscle. J Orthop Traumatol. 2011;12:115118.

30. Levy BA, Dajani KA, Whelan DB, Stannard JP, Fanelli GC, Stuart MJ, et al. Decision making in the multiligament-injured knee: an evidence-based systematic review. Arthroscopy. 2009;25:430-438.

31. Kuroda R, Muratsu H, Harada T, Hino T, Takayama H, Miwa $\mathrm{M}$, et al. Avulsion fracture of the posterior oblique ligament associated with acute tear of the medial collateral ligament. Arthroscopy. 2003,19: E18.

32. Durakbaşa MO, Ulkü K, Ermiş MN. Irreducible open posterolateral knee dislocation due to medial meniscus interposition. Acta Orthop Traumatol Turc. 2011;45:382-386.

33. Chuang TY, Ho WP, Hsieh PH, Yu SW, Chen YJ, Chen CH. One-stage posterior cruciate ligament inlay reconstruction combining anterior cruciate ligament reconstruction following knee dislocation. Arthroscopy. 2006;22:339.e1-7. 